\title{
XXIV. The form of Newton's Rings
}

\section{A. W. Flux B.A.}

To cite this article: A. W. Flux B.A. (1890) XXIV. The form of Newton's Rings, Philosophical Magazine Series 5, 29:178, 217-243, DOI: 10.1080/14786449008619931

To link to this article: http://dx.doi.org/10.1080/14786449008619931

$$
\text { 曲 Published online: } 08 \text { May } 2009 .
$$

Submit your article to this journal 준

LII Article views: 3

Q View related articles $\asymp$ 
LONDON, EDINBURGH, AND DUBLIN

\title{
PHILOSOPHICAL MAGAZINE
}

\author{
AND \\ JOURNAL OF SCIENCE.
}

[FIFTH SERIES ]

$M A R C H \quad 1890$

XXIV. The Form of Newton's Rings. By A. W. FuUx, B.A., Fellow of St. John's College, Cumbridge*.

THE following essay deals with the formation of the equa1 tions of Newton's Rings, when the inclination of the surfaces concerned in their formation is taken into account, not merely with regard to its effects in producing a gradually varying thickness of the intervening medium, but also in deflecting the rays so that, though parallel at incidence, they are no longer so on emergence.

Owing chiefly to the finite size of the sources of light available, in spite of the use of the best collimating instruments, the incident light is not a single plane wave, but consists of an infinite series of such waves having their fronts inclined at small angles. Each incident wave will produce a series of colour-effects at any point in the field of view, but these are in general masked by the effects due to other waves; only in the neighbourhood of a certain surface does there exist distinct coloration. The main result of the calculations of this paper is the determination of the surface on which the colour-effects are most clearly defined. This bas been effected both for reflected and for transmitted light.

The conclusions arrived at are the following :-

(1) The rings lie on a certain ruled surface of the third order, which cuts the planes through the central spot parallel

* Communicated by the Author.

Phil. Mag. S. 5. Vol. 29. No. 178. March 1890. S 
and perpendicular to the plane of incidence, in two straight lines, which cross each other without intersecting.

We shall refer to these lines as the "Principal" line and the "Transverse" line.

(2) The rings are the curves in which this ruled surface is intersected by a set of coaxial cylinders, which are oblique cylinders on circular bases.

In the case of the transmitted system the rings are not rigidly coaxial, though for ordinary angles of incidence the defect could only be detected by means of very refined instruments.

(3) The rings are symmetrical with regard to the first of the two planes mentioned in (1), both in shape and distinctness.

(4) The principal line being inclined to the surfaces of the plate of glass used in producing the rings, after the ring of a determinate order, every ring will lie partially above the upper surface of the plate. Similarly every ring beyond another determinate limit will lie partly below the lower surface.

(5) The points of the principal line are always clearly defined. In all other directions the clearness varies with the incidence, decreasing as the angle of incidence increases, in general.

Every ring whose order does not exceed a certain limit, determinate for each angle of incidence, will have, however, a pair of points quite clearly defined. These points lie symmetrically on the side of the rings furthest from the incident light. Further, the rings are always more clearly defined on this side than on the other.

(6) Any point in a certain line intersecting the principal and transverse lines, and lying between these lines, may be taken as the central spot, which is therefore, to that extent, indeterminate.

The analytical methods of this paper are, for the most part, derived from an article in Band xii. of Wiedemann's Annalen der Physik und Chemie, by Herr Wangerin. Experimental evidence supporting the theory may be found in the first article in that volume.

The results attained by Wangerin are in all respects identical with those of this paper. I have endeavoured to profit by some changes in Herr Wangerin's method, developed in later articles, and to modify these and combine them so as to derive a method which seemed to me to be satisfactory.

The results for transmitted light have been given by Herr Gumlich in Band xxvi. of Wiedemann's Annalen. 


\section{Part I.-Newton's Rings in Reflected Light.}

Section I. Calculation of the Relative Retardations.

Suppose a plate of plane parallel glass to be in contact at the point $O$ (fig. 1 ) with the surface of a lens, whose upper surface is convex and of radius $r$.

Fig. 1.

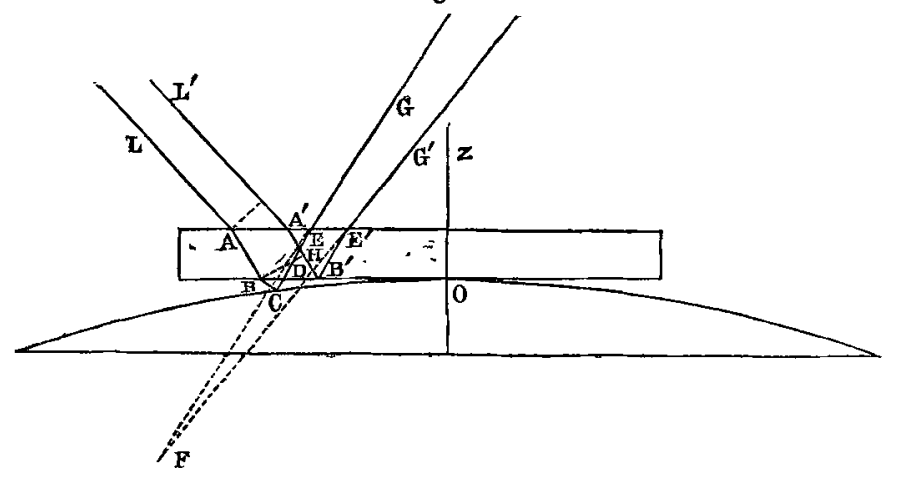

Let $d$ be the thickness of the glass, $\mu$ its refractive index.

Let $L A B C D E G$ be the course of a ray incident on the plate at $A$ and reflected at the lens at $C$.

Let $L^{\prime} A^{\prime} B^{\prime} E^{\prime} G^{\prime}$ be the course of a ray belonging to the same incident wave-front and reflected at the lower face of the plate at $\mathbf{B}^{\prime}$.

Suppose the pair of rays so chosen that their directions after emergence intersect at the point $\mathrm{F}$.

Let the interval between plate and lens be supposed to contain the same medium (air) as the space above the plate.

Let $O$ be taken as origin, the common normal to lens and plate, drawn on the side towards the plate, as axis of $z$, and let axes of $x$ and $y$ be taken in the lower face of the plate.

Take

Let

$\begin{array}{llll}\xi, \eta, \zeta & \text { as coordinates of } \mathrm{F} . \\ \xi_{1}, \eta_{1}, \zeta_{1} & " & " & \text { C. } \\ \xi_{2}, \eta_{2}, \zeta_{2} & " & " & \text { B. } \\ u, v, 0 & \# & " & \text { B }^{\prime} .\end{array}$

$l, m, n$ be the direction-cosines of AL or $\mathrm{CB}$,

$l_{1}, m_{1}, n_{1} \quad " \quad " \quad$ BA or $\mathrm{B}^{\prime} \mathrm{A}^{\prime}$,

$l^{\prime}, m^{\prime}, n^{\prime} \quad " \quad " \quad " \quad \mathrm{CD}$,

$l_{1}{ }^{\prime}, m_{1}{ }^{\prime}, n_{1}{ }^{\prime} \quad$ " $\quad$ " DE.

S 2 
Then

$$
\begin{aligned}
& -l_{1},-m_{1}, n_{1} \text { will be the direction-cosines of } \mathrm{B}^{\prime} \mathrm{E}^{\prime} \text {. } \\
& \begin{array}{ccccc}
-l,-m, n & " & n & " & \mathbf{E}^{\prime} G^{\prime}, \\
l^{\prime}, m^{\prime}, n^{\prime} & " & n & n & \mathbf{E G} .
\end{array}
\end{aligned}
$$

If we refer $\mathrm{C}$ to spherical coordinates with the radius through $O$ as polar axis, $\phi, \psi$ being those coordinates,

$$
\left.\begin{array}{l}
\xi_{1}=r \sin \phi \cos \psi, \quad \eta_{1}=r \sin \phi \sin \psi . \\
\zeta_{1}=-r+r \cos \phi=-2 r \sin ^{2} \frac{\phi}{2}=-\frac{r \phi^{2}}{2}, q \cdot p .
\end{array}\right\} .
$$

The normal at $\mathrm{C}$ has direction-cosines,

$$
\begin{gathered}
\sin \phi \cos \psi, \quad \sin \phi \sin \psi, \quad \cos \phi . \\
\therefore \quad l^{\prime}=-l+2 U \sin \phi \cos \psi, \\
m^{\prime}=-m+2 U \sin \phi \sin \psi, \\
n^{\prime}=-n+2 U \cos \phi ;
\end{gathered}
$$

where$$
\mathrm{U}=l \sin \phi \cos \psi+m \sin \phi \sin \psi+n \cos \phi
$$$$
=l \phi \cos \psi+m \phi \sin \psi+n\left(1-\frac{\phi^{2}}{2}\right) \text { to the } 2 \text { nd order; }
$$$$
\therefore
$$$$
\left.\begin{array}{l}
l^{\prime}=-l+2 n \phi \cos \psi+2 \phi^{2} \cos \psi[l \cos \psi+m \sin \psi] \\
m^{\prime}=-m+2 n \phi \sin \psi+2 \phi^{2} \sin \psi[l \cos \psi+m \sin \psi] \\
n^{\prime}=n+2 \phi[l \cos \psi+m \sin \psi]-2 n \phi^{2}
\end{array}\right\}
$$

We have also

Whence

$$
\begin{aligned}
& l_{1}=l / \mu, \quad m_{1}=m / \mu, \quad n_{1}=\sqrt{\mu^{2}-1+n^{2}} / \mu, \\
& l_{1}^{\prime}=l^{\prime} / \mu, \quad m_{1}^{\prime}=m^{\prime} / \mu, \quad n_{1}^{\prime}=\sqrt{\mu^{2}-1+n^{\prime 2}} / \mu .
\end{aligned}
$$

$$
\left.\begin{array}{r}
\frac{1}{n_{1}^{\prime}}=\frac{1}{n_{1}}\left[1-\frac{2 n}{\mu^{2} n_{1}}(l \phi \cos \psi+m \phi \sin \psi)+\frac{2 n^{2}}{\mu^{2} n_{1}^{2}} \phi^{2}\right. \\
\left.+\frac{2}{\mu^{2} n_{1}^{2}}\left[\frac{3 n^{2}}{\mu^{2} n_{1}^{2}}-1\right](l \phi \cos \psi+m \phi \sin \psi)^{2}\right] \\
\frac{1}{n^{\prime}}=\frac{1}{n}\left[1-\frac{2}{n}(l \phi \cos \psi+m \phi \sin \psi)+2 \phi^{2}\right. \\
\left.+\frac{4}{n^{2}}(l \phi \cos \psi+m \phi \sin \psi)^{2}\right]
\end{array}\right\}
$$


Again,

where

$$
\begin{aligned}
& u=\xi-\frac{l}{n}(d-\zeta)+\frac{l_{1}}{n_{1}} d=\xi+\frac{l}{n}\left(\zeta-\delta_{1}\right), \\
& v=\eta-\frac{m}{n}(d-\zeta)+\frac{m_{1}}{n_{1}} d=\eta+\frac{m}{n}\left(\zeta-\delta_{1}\right),
\end{aligned}
$$

And

$$
\delta_{1} \equiv d\left[1-\frac{n}{\mu n_{1}}\right] .
$$

$$
\begin{aligned}
& \xi_{1}=\xi+\frac{l^{\prime}}{n^{\prime}}(d-\zeta)-\frac{l_{1}^{\prime}}{n_{1}} d-\frac{l^{\prime}}{n^{\prime}} 2 r \sin ^{2} \frac{\phi}{2}, \\
& \eta_{1}=\eta+\frac{m^{\prime}}{n^{\prime}}(d-\zeta)-\frac{m_{1}^{\prime}}{n_{1}^{\prime}} d-\frac{m^{\prime}}{n^{\prime}} 2 r \sin ^{2} \frac{\phi}{2} .
\end{aligned}
$$

Inserting the above expressions for the various directioncosines in terms of $l, m, n, \phi, \psi$, and writing

$$
d\left[1-\frac{n^{3}}{\mu^{3} n_{1}^{3}}\right] \equiv \delta_{2}
$$

we have, to the 2 nd order of $\phi$,

$$
\begin{array}{r}
\xi_{1}=\xi+\frac{l}{n}\left(\zeta-\delta_{1}\right)-2 \phi \cos \psi\left(\zeta-\delta_{\tau}\right)-\frac{2 l}{n^{2}}\left(\zeta-\delta_{2}\right) \\
(l \phi \cos \psi+m \phi \sin \psi)+\frac{l}{n} 2 r \sin ^{2} \frac{\phi}{2} \\
=u-2 \phi \cos \psi\left(\zeta-\delta_{1}\right)-\frac{2 l}{n^{2}}(l \phi \cos \psi+m \phi \sin \psi) \\
\left(\zeta-\delta_{2}\right)+\frac{1}{2} \frac{l}{n} r \phi^{2} ;
\end{array}
$$

$\eta_{\mathrm{l}}=v-2 \phi \sin \psi\left(\zeta-\delta_{1}\right)-\frac{2 m}{n^{2}}(l \phi \cos \psi+m \phi \sin \psi)$

$$
\left(\zeta-\delta_{2}\right)+\frac{1}{2} \frac{m}{n} r \phi^{2}
$$

Comparing these values with those in equations (i.), we find that, to the first order,

$$
r \phi \cos \psi=u, \quad r \phi \sin \psi=v . . . .
$$

Using this approximation in the terms of 2 nd order, 
$\left.\begin{array}{l}\phi \cos \psi=\frac{u}{r}-\frac{2 u}{r^{2}}\left(\zeta-\delta_{1}\right)-\frac{2 l}{n^{2}} \frac{l u+m v}{r^{2}}\left(\zeta-\delta_{2}\right)+\frac{1}{2} \frac{l}{n} \frac{u^{2}+v^{2}}{r^{2}}, \\ \left.\phi \sin \psi=\frac{v}{r}-\frac{2 v}{r^{2}}\left(\zeta-\delta_{1}\right)-\frac{2 m}{n^{2}} \frac{l u+m v}{r^{2}}\left(\zeta-\delta_{2}\right)+\frac{1}{2} \frac{m}{n} \frac{u^{2}+v^{2}}{r^{2}} \cdot\right\}\end{array}\right\}$

Also, from the figure,

$$
\xi_{2}=\xi_{1}+\frac{1}{2} \frac{l}{n} r \phi^{2}, \quad \eta_{2}=\eta_{1}+\frac{1}{2} \frac{m}{n} r \phi^{2} . \text {. . (vii.) }
$$

Now, if a plane be drawn through $B$, perpendicular to $B A$ and $B^{\prime} A^{\prime}$ and cutting the latter in $H$, the vibration in the two rays will be in the same phase at $B$ and $H$.

If, then, $\Delta$ denote the retardation of phase at $F$ of the vibration in $\mathrm{FE}$, relative to that in $\mathrm{FE}^{\prime}$,

$$
\begin{aligned}
\Delta & =\mathrm{BC}+\mathrm{CD}+\mu \mathrm{DE}-\mathrm{EF}-\left[\mu \mathrm{HB}^{\prime}+\mu \mathrm{B}^{\prime} \mathrm{E}^{\prime}-\mathrm{E}^{\prime} \mathrm{F}^{\prime}\right] \\
& =2 r \sin ^{2} \frac{\phi}{2}\left[\frac{1}{n}+\frac{1}{n^{\prime}}\right]+\mu d\left[\frac{1}{n_{1}},-\frac{1}{n_{1}}\right]+(d-\zeta)\left[\frac{1}{n}-\frac{1}{n^{\prime}}\right]-\mu \mathrm{HB} \mathrm{B}^{\prime} .
\end{aligned}
$$

Now

$$
\begin{aligned}
\mu \mathrm{BH}^{\prime} & =\mu\left[l_{1}\left(\xi_{2}-u\right)+m_{1}\left(\eta_{2}-v\right)\right] \\
& =l\left[\xi_{1}-u+\frac{1}{2} \frac{l}{n} r^{2} \phi^{2}\right]+m\left[\eta_{1}-v+\frac{1}{2} \frac{m}{n} r \phi^{2}\right] .
\end{aligned}
$$

Inserting the values of $\xi_{1}$ and $\eta_{1}$ in (iv.) and using in (viii.) the values in (iii.) of $\frac{1}{n_{1}}$ and $\frac{1}{n_{1}^{\prime}}$, and the values of $\phi \cos \psi$, $\phi \sin \psi$ in (vi.), we deduce from (viii.) the following:-

$$
\left.\begin{array}{r}
\Delta=n r \phi^{2}+2 n\left(\zeta-\delta_{1}\right) \frac{u^{2}+v^{2}}{r^{2}}+\frac{2}{n}\left[\frac{l u+m v}{r}\right]^{2}\left(\zeta-\delta_{2}\right) \\
=n \frac{u^{2}+v^{2}}{r}-2 n\left(\zeta-\delta_{1}\right) \frac{u^{2}+v^{2}}{r^{2}}-\frac{2}{n}\left[\frac{l u+m v}{r}\right]^{2}\left(\zeta-\delta_{2}\right) \\
+(l u+m v) \frac{u^{2}+v^{2}}{r^{2}} .
\end{array}\right\}
$$

Correct to the third order, inclusive.

This is the result obtained in equation (8) p. 211, Band xii. of the Annalen der Physik.

Section II. The same in the general case.

Before proceeding to the use of the evaluation of the retardation in Sect. I., we shall determine in a similar manner 
theretardation when one of the rays $A B \mathrm{C} \mathrm{B}_{1} \mathrm{C}_{1} \ldots$ $\mathrm{C}_{k-1}$ D E G (fig. 2) is reflected $k$ times at the surface of the

Fig. 2.

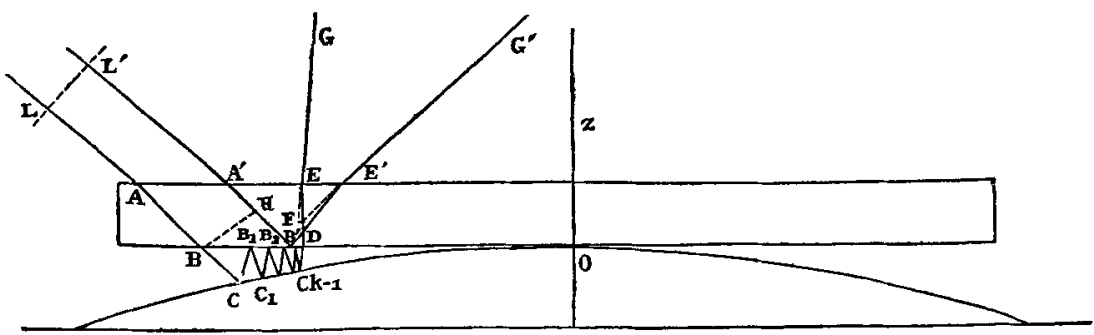

lens, and $\overline{k-1}$ times at the lower surface of the plate, the other ray being only reflected at $\mathrm{B}^{\prime}$ as before.

Take now

$$
\begin{array}{lll}
\xi, \eta, \zeta \text { as coordinates of } \mathrm{F}, \\
\xi_{r}, \eta_{r}, \zeta_{r} & \Rightarrow & \text { of } \mathrm{C}_{r-1}[r<k], \\
\xi, \eta^{\prime}, \zeta & \Rightarrow & \text { of } \mathrm{B}^{\prime}, \\
u, v, 0 \quad & \text { of } \mathrm{B}^{\prime} .
\end{array}
$$

Let the spherical coordinates of $\mathrm{C}, \mathrm{C}_{1} \ldots \mathrm{C}_{r} \ldots$ be $\phi, \psi$; $\phi_{1}, \psi_{1} \ldots ; \phi_{r}, \psi_{r} \ldots$; and let Then

$l_{r}, m_{r}, n_{r}$ be the direction-cosines of $\mathrm{C}_{r-1} \mathrm{~B}_{r}$.

Let

$$
-l r,-m_{r}, n_{r} \text { are the } \quad " \quad \text { of } \mathrm{O}_{r} \mathrm{~B} \text {. }
$$

$l^{\prime}, \quad m^{\prime}, \quad n^{\prime}$ be the direction-cosines of $\mathrm{BA}$ and $\mathrm{B}^{\prime} \mathrm{A}^{\prime}$;

$-l^{\prime},-m^{\prime}, n^{\prime}$ are those of $\mathrm{B}^{\prime} \mathrm{E}^{\prime}$.

$l_{k}^{\prime}, \quad m_{k}^{\prime}, n_{k}^{\prime} \quad$ of DE.

$-l,-m, \quad n \quad, \quad$ of $\mathrm{FG}^{\prime}$.

Also

$$
l_{k}, m_{k}, n_{k} \quad \text {, of FG. }
$$

$$
l_{k}=\mu l_{k}{ }^{\prime}, \quad m_{k}=\mu m_{k}{ }^{\prime}, \quad \sqrt{\mu^{2}-1+n_{k}{ }^{2}}=\mu n_{k}{ }^{\prime} \text {. }
$$

If we write down the coordinates of $B_{1}$, deriving them from the coordinates of $\mathrm{C}$ first, and then from those of $\mathrm{C}_{1}$, we obtain

$$
\begin{aligned}
& r \sin \phi \cos \psi+\frac{l_{1}}{n_{1}} 2 r \sin ^{2} \frac{\phi}{2}=r \sin \phi_{1} \cos \psi_{1}-\frac{l_{1}}{n_{1}} 2 r \sin ^{2} \frac{\phi_{1}}{2}, \\
& r \sin \phi \sin \psi+\frac{m_{1}}{n_{1}} 2 r \sin ^{2} \frac{\phi}{2}=r \sin \phi_{1} \sin \psi_{1}-\frac{m_{1}}{n_{1}} 2 r \sin ^{2} \frac{\phi_{1}}{2} .
\end{aligned}
$$


$\therefore$ To the first order, $\phi \cos \psi=\phi_{1} \cos \psi_{1}, \quad \phi \sin \psi=\phi_{1} \sin \psi_{1}$.

And therefore to the second order,

$$
\begin{aligned}
& \phi_{1} \cos \psi_{1}=\phi \cos \psi-\frac{l}{n} r \phi^{2}, \\
& \phi_{1} \sin \psi_{1}=\phi \sin \psi-\frac{m}{n} r \phi^{2} .
\end{aligned}
$$

Similarly, we have

$$
\begin{aligned}
& \phi_{2} \cos \psi_{2}=\phi_{1} \cos \psi_{1}+\frac{l_{1}}{n_{1}} \phi_{1}{ }^{2}=\phi \cos \psi-2 \frac{l}{n} \phi^{2}, \\
& \phi_{2} \sin \psi_{2}=\phi_{1} \sin \psi_{1}+\frac{m_{1}}{n_{1}} \phi_{1}{ }^{2}=\phi \sin \psi-2 \frac{n}{n} \phi^{2} .
\end{aligned}
$$

And, generally,

$$
\left.\begin{array}{l}
\phi_{k} \cos \psi_{k}=\phi \cos \psi-k \cdot \frac{l}{n} \phi^{2} \\
\phi_{k} \sin \psi_{k}=\phi \sin \psi-k \cdot \frac{m}{n} \phi^{2}
\end{array}\right\} \quad \cdot
$$

Recalling the results of (ii.) section I., we have :-

$$
\begin{aligned}
l_{2} & =l_{1}+2 n_{1}\left[\phi \cos \psi-\frac{l}{n} \phi^{2}\right]-2 \phi_{1}{ }^{2} \cos \psi\left[l_{1} \cos \psi+m_{1} \sin \psi\right] \\
& =-l+4 n \phi \cos \psi-2 l \phi^{2}+8 \phi \cos \psi[l \phi \cos \psi+m \phi \sin \psi] ; \\
m_{2} & =-m+4 n \phi \sin \psi-2 m \phi^{2}+8 \phi \sin \psi[l \phi \cos \psi+m \phi \sin \psi] ; \\
n_{2} & =n+4[l \phi \cos \psi+m \phi \sin \psi]-8 n \phi^{2}-2 \frac{1-n^{2}}{n} \phi^{2} .
\end{aligned}
$$

Proceeding thus, we find that

$$
\left.\begin{array}{c}
l_{k}=-l+2 k n \phi \cos \psi-k(k-1) l \phi^{2} \\
+2 k^{2} \phi \cos \psi[l \phi \cos \psi+m \phi \sin \psi] \\
m_{k}=-m+2 k n \phi \sin \psi-k(k-1) m \phi^{2} \\
+2 k^{2} \phi \sin \psi[l \phi \cos \psi+m \phi \sin \psi] \\
n_{k}=n+2 k[l \phi \cos \psi+m \phi \sin \psi]-2 k^{2} n \phi^{2} \\
-k(k-1) \frac{1-n^{2}}{n} \phi^{2}+2 \frac{k^{2}}{n}[l \phi \cos \psi+m \phi \sin \psi]^{2}
\end{array}\right\}
$$


Form of Newton's Rings.

Whence

$$
\left.\begin{array}{rl}
\frac{1}{n_{b}}= & \frac{1}{n}\left[1-\frac{2 k}{n}(l \phi \cos \psi+m \phi \sin \psi)+2 k^{2} \phi^{2}\right. \\
& \left.+k(k-1) \frac{1-n^{2}}{n^{2}} \phi^{2}+\frac{2 k^{2}}{n^{2}}(l \phi \cos \psi+m \phi \sin \psi)^{2}\right] ; \\
\frac{1}{n_{\grave{k}}^{\prime \prime}}= & \frac{1}{n^{\prime}}\left[1-\frac{2 n k}{\mu^{2} n^{\prime 2}}(l \phi \cos \psi+m \phi \sin \psi)+\frac{2 n^{2}}{\mu^{2} n^{\prime}} k^{2} \phi^{2}\right. \\
& \left.+\frac{1-n^{2}}{\mu^{2} n^{\prime 2}} k(k-1) \phi^{2}+\frac{2 k^{2}}{\mu^{2} n^{\prime 2}}\left(\frac{3 n^{2}}{\mu^{2} n^{\prime 2}}-1\right)(l \phi \cos \psi+m \phi \sin \psi)^{2}\right] .
\end{array}\right\}
$$

Also, from the coordinates of $\mathrm{C}_{k-1}$,

$r \sin \phi_{k-1} \cos \psi_{k-1}=\xi+\frac{l_{k}}{n_{k}}(d-\zeta)-\frac{l_{k}^{\prime}}{n_{k}^{\prime}} d-\frac{l_{k}}{n_{k}} 2 r \sin ^{2} \frac{\phi_{k-1}}{2} ;$

$r \sin \phi_{k-1} \sin \psi_{k-1}=\eta+\frac{m_{k}}{n_{k}}(d-\zeta)-\frac{m_{k}{ }^{\prime}}{n_{k}} d-\frac{m_{k}}{n_{k}} 2 r \sin ^{2} \frac{\phi_{k-1}}{2}$.

Whence, by means of (i.), (ii.), and (iii.),

$$
\left.\begin{array}{c}
\phi \cos \psi=\frac{u}{r}-2 k \frac{u}{r^{2}}\left(\zeta-\delta_{1}\right)-\frac{2 k}{n^{2} r^{2}} l(l u+m v)\left(\zeta-\delta_{2}\right)+\left(k-\frac{1}{2}\right) \frac{l}{n} \frac{u^{2}+v^{2}}{r^{2}} ; \\
\phi \sin \psi=\frac{v}{r}-2 k \frac{v}{r^{2}}\left(\zeta-\delta_{1}\right)-\frac{2 k}{n^{2} r^{2}} m(l u+m v)\left(\zeta-\delta_{2}\right)+\left(k-\frac{1}{2}\right) \frac{m}{n} \frac{u^{2}+v^{2}}{r^{2}}
\end{array}\right\}
$$

We have also

$$
\begin{aligned}
\xi^{\prime}=\xi+\frac{l_{k}}{n_{k}}(d-\zeta) & -\frac{l_{k}{ }^{\prime}}{m_{k}} d+\frac{r}{2}\left[\phi^{2}\left(\frac{l}{n}-\frac{l_{1}}{n_{1}}\right)\right. \\
& \left.+\phi_{1}{ }^{2}\left(-\frac{l_{1}}{n_{1}}-\frac{l_{2}}{n_{2}}\right)+\ldots \text { to } k \text { terms }\right] \\
\eta^{\prime}=\eta+\frac{m_{k}}{n_{k}}(d-\zeta) & -\frac{m_{k}{ }^{\prime}}{n_{k}{ }^{\prime}} d+\frac{r}{2}\left[\phi^{2}\left(\frac{m}{n}-\frac{m_{1}}{n_{1}}\right)\right. \\
& \left.+\phi_{1}{ }^{2}\left(-\frac{m_{1}}{n_{1}}-\frac{m_{2}}{n_{2}}\right)+\ldots \text { to } k \text { terms }\right] .
\end{aligned}
$$

Drawing the plane $\mathrm{BH}$, perpendicular to $\mathrm{BA}$ and $\mathrm{B}^{\prime} \mathrm{A}^{\prime}$ as before, the retardation at $F$ of the vibration in $F E$ relative to that in $\mathrm{FE}^{\prime}$ is

$$
\Delta_{k}=\mathrm{BC}+\mathrm{CB}_{1}+\ldots+\mathrm{C}_{k-1} \mathrm{D}+\mu d\left[\frac{1}{n_{k}^{\prime}}-\frac{1}{n^{\prime}}\right]+(d-\zeta)\left[\frac{1}{n}-\frac{1}{n_{k}}\right]-\mu \mathrm{B}^{\prime} \mathrm{H} \text {. }
$$

And

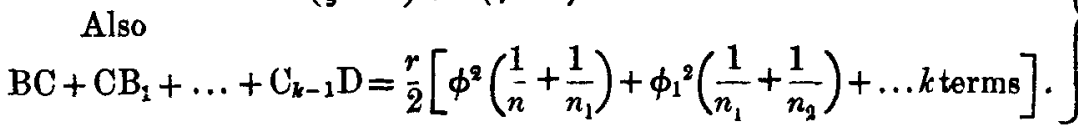

$$
\begin{aligned}
\mu \cdot \mathrm{B}^{\prime} \mathrm{H} & =\mu l_{1}\left(\xi^{\prime}-u\right)+\mu m_{1}\left(\eta^{\prime}-v\right) \\
& =l\left(\xi^{\prime}-u\right)+m\left(\eta^{\prime}-v\right)
\end{aligned}
$$


Using the values obtained in (ii.), (iii.), (iv.), (v.), (vii.) in evaluating $\Delta$ in (vi.) we obtain

$$
\left.\begin{array}{r}
\Delta_{k}=n r k \phi^{2}+2 k^{2} n \frac{u^{2}+v^{2}}{r^{2}}\left(\zeta-\delta_{1}\right)+\frac{2 k^{2}}{n}\left(\frac{l u+m v}{r}\right)^{2}\left(\zeta-\delta_{2}\right) \\
-k(k-1) \frac{u^{2}+v^{2}}{r^{2}}(l u+m v), \\
=k n \frac{u^{2}+v^{2}}{r}-k^{2}\left[2 n \frac{u^{2}+v^{2}}{r^{2}}\left(\zeta-\delta_{1}\right)\right. \\
\left.+\frac{2}{n}\left(\frac{l u+m v}{r}\right)^{2}\left(\zeta-\delta_{2}\right)-(l u+m v) \frac{u^{2}+v^{2}}{r^{2}}\right] .
\end{array}\right\}
$$

Section III. Determination of the Equations of the Rings.

If w' $\theta$ now determine the axes of $x$ and $y$ so that the plane $x z$ is parallel to the incident light, and if $\theta$ be the angle of incidence, Also

$$
l=\sin \theta, \quad m=0, \quad n=\cos \theta .
$$

where

$$
\delta_{1}=d\left[1-\frac{\cos \theta}{\mu \cos \theta_{1}}\right], \quad \delta_{2}=d\left[1-\frac{\cos ^{3} \theta}{\mu^{3} \cos ^{3} \theta_{1}}\right],
$$

$$
\sin \theta=\mu \sin \theta_{1}, u=\xi+\tan \theta\left(\zeta-\delta_{1}\right), \quad v=\eta .
$$

And with this simplification

$$
\begin{aligned}
\Delta_{k}=k \cos \theta \frac{u^{2}+v^{2}}{r} & -\frac{k^{2} \cos \theta}{r^{2}}\left[2\left(u^{2}+v^{2}\right)\left(\zeta-\delta_{1}\right)\right. \\
& \left.+2 u^{2} \tan ^{2} \theta\left(\zeta-\delta_{2}\right)-u \tan \theta\left(u^{2}+v^{2}\right)\right] .
\end{aligned}
$$

We shall find it convenient to refer the system to a new origin and new axis of $z$, keeping the axis of $x$ and $y$ parallel to the directions just chosen.

The new axis of $z$ is inclined at an angle $\theta$ to its old direction, i.e. is parallel to the emergent light which has been but once reflected at the surface of the plate. The new origin is the point $\left(\xi_{0} \zeta_{0}\right)$, where

$$
\xi_{0}=-d\left(\mu^{2}-1\right) \tan ^{3} \theta_{1}, \quad \xi_{0}=d\left[1-\frac{\cos ^{3} \theta}{\mu \cos ^{3} \theta_{1}}\right] .
$$

The formulæ of transformation are :-

$$
\begin{aligned}
& \xi=\xi_{0}+x-z \sin \theta, \\
& \eta=y, \quad \text { (See fig. 3.) } \\
& \zeta=\zeta_{0}+z \cos \theta . \quad
\end{aligned}
$$


Fig. 3.

We shall also write

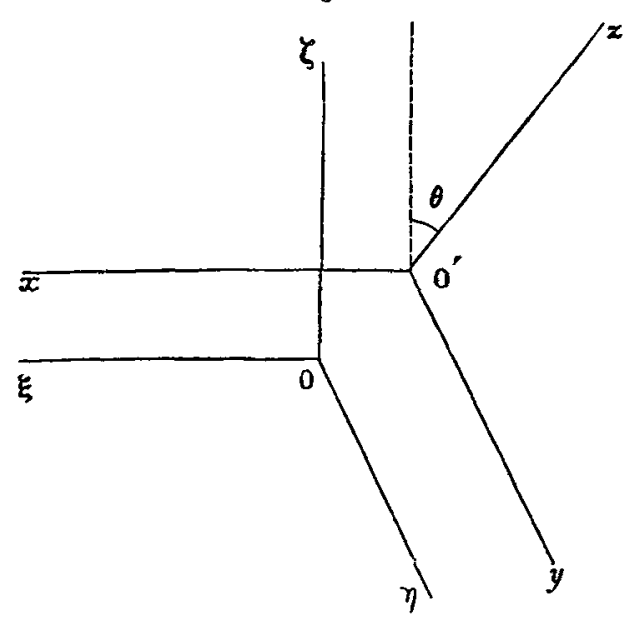

$$
\begin{aligned}
& z_{1} \cos \theta=\left(\delta_{2}-\delta_{1}\right) \sin ^{2} \theta=d \frac{\mu^{2}-1}{\mu^{3} \cos ^{3} \theta_{1}} \cos \theta \sin ^{2} \theta ; \\
& \text { i.e. } z_{1}=\frac{\mu^{2}-1}{\mu^{3} \cos ^{3} \theta_{1}} d \sin ^{2} \theta=d \frac{\left(\mu^{2}-1\right) \tan ^{2} \theta_{1}}{\mu \cos \theta_{1}} .
\end{aligned}
$$

With the assistance of this change of symbols, we shall consider the retardations of systems of incident rays slightly inclined to the incident rays hitherto considered.

If $\phi$ be the angle between such ravs ( $\phi$ being small) and $\psi$ the angle which the plane through the two rays makes with the plane $x z$, we have

$$
\left.\left.\begin{array}{c}
l=\sin \theta-\cos \theta \cdot \phi \cos \psi, \\
m=\phi \sin \psi, \\
n=\cos \theta+\sin \theta \cdot \phi \cos \psi .
\end{array}\right\} . . . \text { (v. }\right)
$$


$\therefore$ The new value of $\delta_{1}$,

$$
\begin{aligned}
& \delta_{1}^{\prime}=d\left[1-\frac{n}{\mu n_{1}}\right] \text {, } \\
& =d\left[1-\frac{\cos \theta}{\mu \cos \theta_{1}}(1+\tan \theta \cdot \phi \cos \psi)\left(1-\frac{\sin \theta \cos \theta}{\mu^{2} \cos ^{2} \theta_{1}} \phi \cos \psi\right)\right] \text {, } \\
& =d\left[1-\frac{\cos \theta}{\mu \cos \theta_{1}}\right]-d \frac{\cos \theta}{\mu \cos \theta_{1}} \phi \cos \psi \tan \theta-\left[\frac{\sin \theta \cos \theta}{\mu^{2} \cos ^{2} \theta_{1}}\right], \\
& =\delta_{1}-d \frac{\sin \theta\left(\mu^{2}-1\right)}{\mu^{3} \cos ^{3} \theta_{1}} \phi \cos \psi \text {, } \\
& \begin{array}{l}
=\delta_{1}-\frac{z_{1}}{\sin \theta} \phi \cos \psi \text {. . . . . . . } \\
+\frac{l}{n}\left(\zeta-\delta_{1}^{\prime}\right), \\
\xi_{0}+x-z \sin \theta+\tan \theta\left(1-\frac{\phi \cos \psi}{\sin \theta \cos \theta}\right)
\end{array} \\
& \left(\zeta_{0}+z \cos \theta-\delta_{1}+z_{1} / \sin \theta \cdot \phi \cos \psi\right), \\
& =x+\phi \cos \psi\left[\frac{z_{1}}{\sin \theta}-\frac{z}{\sin \theta}-\frac{d\left(\mu^{2}-1\right) \tan ^{3} \theta_{1}}{\sin ^{2} \theta}\right] \tan \theta, \\
& =x-\frac{z}{\cos \theta} \phi \cos \psi \text {. . . . . . . . . . . . . (vii.) } \\
& v=\eta+\frac{m}{n}\left(\zeta-\delta_{1}{ }^{\prime}\right), \\
& =y+\frac{\phi \sin \psi}{\cos \theta}\left(z+z_{1}\right) \cos \theta=y+\left(z+z_{1}\right) \phi \sin \psi \text {. (viii.) }
\end{aligned}
$$

Therefore

$\Delta_{k}^{\prime}=\frac{k}{r}(\cos \theta+\sin \theta \cdot \phi \cos \psi)\left[x^{2}+y^{2}\right.$

$$
\begin{aligned}
\left.-\frac{2 x z}{\cos \theta} \phi \cos \psi+2 y\left(z+z_{1}\right) \phi \sin \psi\right] \\
-\frac{k^{2} \cos ^{2} \theta}{r^{2}}\left[2\left(x^{2}+y^{2}\right)\left(z+z_{1}\right)\right. \\
\left.+2 x \tan ^{2} \theta\left(z-z_{1} \cot ^{2} \theta\right)-\frac{x \tan \theta}{\cos \theta}\left(x^{2}+y^{2}\right)\right]
\end{aligned}
$$

to the 3rd order of small quantities, $x, y, z, z_{1}, \phi$ bejng of the 1st order;

$$
\begin{aligned}
&= \Delta k+\frac{k}{r}\left(\left(x^{2}+y^{2}\right) \sin \theta-2 x z\right) \phi \cos \psi \\
&+\frac{k}{r} \cdot 2 y\left(z+z_{1}\right) \cos \theta \cdot \phi \sin \psi, \\
&=\mathrm{A}+\mathrm{B} \phi \cos \psi+\mathrm{C} \cdot \phi \sin \psi \text { (say). . . . . (ix.) }
\end{aligned}
$$


This expression varies least from its mean value $A$, as $\phi$ and $\psi$ vary, the former from zero to a small value, the latter from 0 to $2 \pi$, when $\mathrm{B}^{2}+\mathrm{C}^{2}$ is least, $i$. $e$. when

i.e.

$$
\mathrm{B} \cdot \frac{\partial \mathrm{B}}{\partial z}+\mathrm{C} \cdot \frac{\partial \mathrm{C}}{\partial z}=0 \text {, }
$$

$$
2 x\left[\left(x^{2}+y^{2}\right) \sin \theta-2 x z\right]-4 y^{2} \cos ^{2} \theta\left(z+z_{1}\right)=0,
$$

or

$$
x\left(x^{2}+y^{2}\right) \sin \theta-2 x^{2} z-2 y^{2} \cos ^{2} \theta\left(z+z_{1}\right)=0,
$$

or

$$
\begin{array}{r}
2\left(x^{2}+y^{2}\right)\left(z+z_{1}\right) \cos ^{2} \theta+2 x^{2} \sin ^{2} \theta\left(z-z_{1} \cot ^{2} \theta\right) \\
-x \sin \theta\left(x^{2}+y^{2}\right)=0 .
\end{array}
$$

When (x.) holds, the value of $\Delta_{k}$ in (i.) becomes

$$
\Delta_{k}=\frac{k}{r} \cos \theta\left(u^{2}+v^{2}\right)
$$

i. e. the $\mathrm{A}$ of equation (ix.)

$=k \cos \theta \frac{x^{2}+y^{2}}{r}=k \delta$, where $\delta=\frac{\cos \theta}{r}\left(x^{2}+y^{2}\right) . .$.

With this value, corresponding closely with that given in the ordinary theory of Newton's Rings, we can determine the equations of those rings at once. The ordinary methods used for thin plates by Glazebrook or Airy furnish the result that the equations are (for the dark rings) :-

$$
\frac{\cos \theta}{r}\left(x^{2}+y^{2}\right)=h \lambda \text {, }
$$

i.e.

$$
x^{2}+y^{2}=\frac{h r \lambda}{\cos \theta}=a^{2} \text { (say). . . . . }
$$

i. e. The rings lie on this set of coaxial elliptic cylinders.

They also lie on the surface of the 3 rd order $(x$.$) .$

The principle which we have employed to determine the position of the rings is both simple and satisfactory. Though any pair of intersecting rays received into the eye may be regarded as producing interference-results, yet only such need be considered as have their effect neutralized in the least degree by that of other pairs of interfering rays.

Since, in every case of experiment, the incident light is not a simple plane wave, but consists of many plane waves, only limited in direction by the size of the pupil of the eye or object-glass of the observing-instrument, and therefore in general but little inclined to one another, we shall only be able to observe those colour-effects in which these various waves combine to the most complete extent. 
These lie on the surface (x.), where it is intersected by the series of coaxial elliptic cylinders (oblique cylinders on circular bases) (xii.).

Section IV. The Surface of Interference.

We have now to examine the surface whose equation is given in (x.) of the last section.

We shall use the form

$$
x\left(x^{2}+y^{2}\right) \sin \theta-2 x^{2} z-2 y^{2} \cos ^{2} \theta\left(z+z_{1}\right)=0 .
$$

The points of the surface which we have to consider are its intersections with

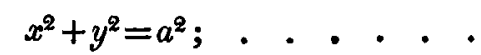

where

$$
a^{2}=\frac{h r \lambda}{\cos \theta} \text { or } \frac{2 h+1}{2} \frac{r \lambda}{\cos \theta},
$$

according as we are considering dark or bright rings. Where (i.) is intersected by $y=0$,

$$
x \sin \theta-2 z=0 \text {. . . . . . }
$$

The intersection is therefore a straight line through the origin.

If $\omega$ be the angle made by this line with the axis of $x$,

$$
\begin{aligned}
& \sin \theta=\frac{2 z}{x}=\frac{2 \sin \omega}{\cos (\theta-\omega)}, \\
\therefore \quad \tan \omega & =\frac{\sin \theta \cos \theta}{1+\cos ^{2} \theta} .
\end{aligned}
$$

Fig. 4.

(See fig. 4.)

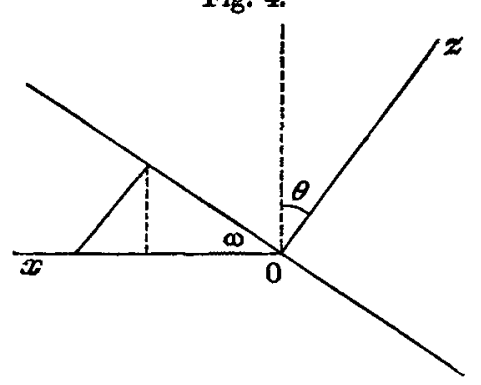

We may notice that when $\theta=0$ and when $\theta=\frac{\pi}{2}, \tan \omega$ vanishes.

It attains a maximum value when $\tan \theta=\sqrt{2}$, 
i.e. when

$$
\theta=54^{\circ} 44^{\prime} \quad 8^{\prime \prime} \cdot 2 \text { (about). }
$$

For this value

$$
\omega=19^{\circ} 28^{\prime} 16^{\prime \prime} \cdot 4 \text { (about). }
$$

This is the greatest value of $\omega$, which varies from zero when $\theta$ is zero to this greatest value, and then decreases to zero again as $\theta$ approaches a right angle. We notice that the value of $\omega$ is entirely independent of the shape, thickness, and material of the lens and plate employed.

The extreme points of any ring in this line are by (ii.) $x= \pm a$.

The actual diameter of the ring is therefore

$$
\begin{gathered}
2 a \frac{\cos \theta}{\cos (\theta-\omega)}=a \frac{\sin \theta \cos \theta}{\sin \omega} \\
\quad=a \sqrt{1+3 \cos ^{2} \theta} . .
\end{gathered}
$$

The diameter, as seen projected on the horizontal, is the distance from $x=a, z=0$, to $x=-a, z=0$, i.e. $2 a$.

Let us now consider the intersection of (i.) with $y= \pm a$.

Since, so far as the rings are concerned, equation (ii.) is also to be considered, so that $x=0$, we see from (i.) that

$$
z=-z_{1} \text {, }
$$

i. $e$. the intersection of this plane with the ring-system is also a straight line, and, moreover, a line parallel to the surfaces of the glass plate.

We may write (i.) in the form

$$
z=\frac{1}{2} \frac{x\left(x^{2}+y^{2}\right) \sin \theta}{x^{2}+y^{2} \cos ^{2} \theta}-z_{1} \frac{y^{2} \cos ^{2} \theta}{x^{2}+y^{2} \cos ^{2} \theta} .
$$

Thus, when $x$ and $y$ vanish, $z$ lies between zero and $-z_{1}$, the exact value depending on the vanishing ratio of $x$ to $y$.

Thus this portion of the axis of $z$ lies entirely in the surface.

Again, we may write the equation (i.) in the form

$$
\left(x^{2}+y^{2}\right)(x \sin \theta-2 z)+2 y^{2} \sin ^{2} \theta\left[z-z_{1} \cot ^{2} \theta\right]=0 ;
$$

and hence we see that the line

$$
\left.\begin{array}{l}
z=z_{1} \cot ^{2} \theta \\
x=\frac{2 z}{\sin \theta}=\frac{2 z_{1} \cos ^{2} \theta}{\sin ^{3} \theta}
\end{array}\right\}
$$

lies wholly in the surface.

This is a line parallel to the axis of $y$. 
If we take a plane

$$
x \sin \theta-2 z+\lambda\left(z-z_{1} \cot ^{2} \theta\right)=0
$$

passing through the line (vii.), it cuts the surface in the pair of straight lines

$$
\frac{y}{x}= \pm \sqrt{\frac{\lambda}{2 \sin ^{2} \theta-\lambda}} .
$$

It cuts the axis of $z$ where

$$
z=z_{1} \cot ^{2} \theta \frac{\lambda}{\lambda-2} \text {. }
$$

Denoting this value of $z$ by $z_{0}$, we see that

$$
\frac{y}{x}= \pm \sec \theta \sqrt{\frac{-z_{0}}{z_{1}+z_{0}}}
$$

gives the pair of lines in which the above plane meets the surface.

It appears, therefore, that the surface is generated by pairs of lines intersecting the axis of $z$ between the origin and $z=-z_{1}$, and also intersecting the line (vii.). If we denote the origin by $\mathrm{P}$ (fig. 5), $z=-z_{1}$ by $\mathrm{Q}$, and $z=-z_{0}$ by $\mathrm{M}$,

\section{Fig. 5.}

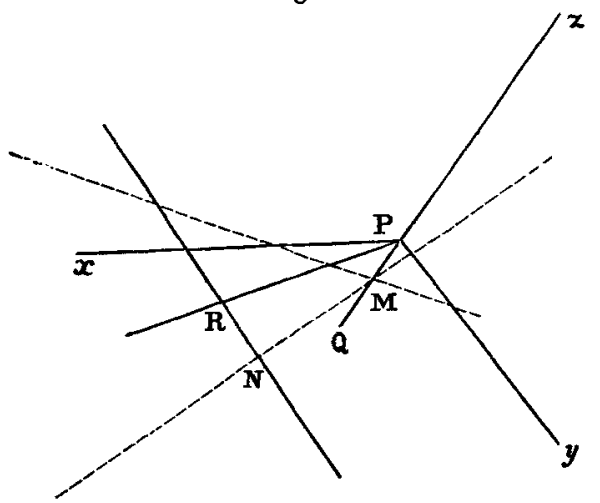

and also the line (vii.) by $\mathrm{RN}, \mathrm{R}$ being its intersection with the plane $x z$, and $\mathrm{N}$ its intersection with one of the above pair of lines,

$$
\begin{aligned}
\mathrm{RN} & =\frac{2 z_{1} \cos ^{2} \theta}{\sin ^{3} \theta} \cdot \pm \sec \theta \sqrt{\frac{\overline{\mathrm{PM}}}{\overline{\mathrm{Q}}}} \\
& = \pm \frac{2 \cos \theta}{\sin ^{3} \theta} \mathrm{PQ} \sqrt{\overline{\mathrm{PM}}},
\end{aligned}
$$

giving the law of the generators. 
Section V. Some Points about the Rings.

From these details concerning the "surface of interference" we may learn something about the rings.

It is evident that since the plane $x z$ cuts the rings in a straight line inclined to the surfaces of the plate, we shall, by proceeding far enough in either direction, at last reach rings which lie partially above or partially below the plate.

The upper surface of the plate is

The lower surface is $z=-z_{1}$.

$$
\begin{aligned}
z & =\left(d-\zeta_{0}\right) \sec \theta \\
& =d \sec \theta-z_{i} .
\end{aligned}
$$

Hence the line (iii.) in the last section intersects the upper surface of the plate where

$$
x=\frac{2\left(d-z_{1} \cos \theta\right)}{\sin \theta \cos \theta}=\frac{2 d \cos ^{2} \theta}{\mu \sin \theta \cos ^{3} \theta_{1}}
$$

and the lower surface where

$$
x=\frac{-2 z_{1}}{\sin \theta}=-2 d \frac{\mu^{2}-1}{\mu^{3} \cos ^{3} \theta_{1}} \sin \theta,
$$

since the order of the ring in this direction $(y=0)$ is given by

$$
\begin{aligned}
& x= \pm a= \pm \sqrt{\frac{\overline{h \lambda r}}{\cos \theta} \text { for the dark rings, }} \\
&= \pm \sqrt{\frac{(2 h+1) \lambda r}{2 \cos \theta} \text { for the bright rings. }} \\
& \text { If } \quad h>\frac{4 d^{2}}{\lambda r} \frac{\cos ^{5} \theta}{\mu^{2} \sin ^{2} \theta \cos ^{6} \theta_{1}},
\end{aligned}
$$

the dark ring of order $h$ has points above the upper surface of the glass plate.

If

$$
h>\frac{4 d^{2}}{\lambda r} \frac{\cos ^{5} \theta}{\mu^{2} \sin ^{2} \theta \cos ^{6} \theta_{1}}-\frac{1}{2},
$$

the bright ring of order $h$ lies partially above the upper surface of the glass plate.

$$
\text { If } \quad h>\frac{4 d^{2}}{\lambda r} \frac{\left(\mu^{2}-1\right)^{2} \sin ^{2} \theta \cos \theta}{\mu^{6} \cos ^{6} \theta_{1}} \text {, }
$$

the dark ring of order $h$ lies partially below the lower surface of the glass plate; and if $h+\frac{1}{2}$ be greater than the quantity Phil. Mag. S. 5. Vol. 29. No. 178. March 1890. 
just written, the bright ring of order $h$ lies partly below the glass plate.

If we denote the line (iii.) of the last section, viz.:

the principal line, and the plane

$$
\left.\begin{array}{rl}
x \sin \theta-2 z & =0 \\
y & =0
\end{array}\right\},
$$

the principal plane.

$$
x \sin \theta-2 z=0,
$$

The rings lie wholly below the prineipal plane so long as

$$
z<\frac{x \sin \theta}{2} \text {. }
$$

The greatest value of $z$ determined from equations (i.) and (ii.) of the last section is less than $\frac{x \sin \theta}{2}$ so long as

i. e.

$$
\begin{aligned}
& a^{2}<4 d^{2} \frac{\left(\mu^{2}-1\right) \cos ^{4} \theta}{\mu^{6} \sin ^{2} \theta \cos ^{6} \theta_{1}} ; \\
& h<\frac{4 d^{2}}{\lambda r} \frac{\left(\mu^{2}-1\right) \cos ^{5} \theta}{\mu^{6} \sin ^{2} \theta \cos ^{6} \theta_{1}} .
\end{aligned}
$$

It will be observed that this value of $h$ and the first two of those just given is greater as $\theta$ is smaller and decreases as $\theta$ increases.

It is otherwise with the number determining the order of ring lying below the lower surface of the glass plate.

It was found that the axis of $z$ lies entirely in the surface between $z=0$ and $z=-z_{1}$.

Thus there arises a certain degree of indeterminateness with regard to the central black spot, any point between these limits being seen with equal clearness. This difficulty is actually found in the course of the experiments undertaken in connexion with this more exact theory of Newton's Rings.

Section VI. Relative Distinctness of Different Portions of the Ring-system.

We shall now consider where and under what conditions the rings are formed most distinctly.

In Sect. III. (ix.) the expression found for the retardation was

$$
\begin{aligned}
\Delta_{k}{ }^{\prime} & =\mathrm{A}+\mathrm{B} \phi \cos \psi+\mathrm{C} \phi \sin \psi, \\
& =\mathrm{A}+\sqrt{\mathrm{B}^{2}+\mathrm{C}^{2}} \phi \cos (\psi-\alpha), \text { where } \tan \alpha=\frac{\mathrm{C}}{\mathrm{B}} .
\end{aligned}
$$

When $\mathrm{B}^{2}+\mathrm{C}^{2}$ is a minimum,

$$
\mathrm{A}=\frac{k}{r} \cos \theta\left(x^{2}+y^{2}\right)
$$


Also

$$
\begin{aligned}
& \mathrm{B}=\frac{k}{r}\left[\left(x^{2}+y^{2}\right) \sin \theta-2 x z\right], \\
& \mathrm{C}=\frac{k}{r} 2 y \cos \theta\left(z+z_{1}\right) .
\end{aligned}
$$

We have hitherto not considered the effect of the term involving $\phi$, but since the minimum of $\mathrm{B}^{2}+\mathrm{C}^{2}$ is not always zero, we cannot pass these terms over without notice.

When $y=0$, and $x \sin \theta-2 z=0, \mathrm{~B}$ and $\mathrm{C}$ ranish, and therefore the points on the line in the plane $x z$ are always formed clearly.

The only reason why we cannot see rings of a very high order in this plane is that the rings of different colour's overlap to such an extent as to obscure the result if the incident light be not strictly monochromatic. fact,

When $x=0$, and $z=-z_{1}$, $C$ vanishes, but $B$ does not; in

$$
\begin{aligned}
\mathrm{B} & =\frac{k}{r} y^{2} \sin \theta, \\
& =\frac{k}{r} h r \lambda \tan \theta ;
\end{aligned}
$$

since $y^{2}=a^{2}=\frac{l r \lambda}{\cos \theta}$, when $x=0$.

Therefore, the distinctness depends on the magnitude of $h \lambda \tan \theta$. This quantity increases as $h$ increases, and therefore the outer rings in the direction of the axis of $y$ are always less distinct than the inner. Further, as $\theta$ increases, $\tan \theta$ increases; and when the incidence is nearly grazing, very few rings can be formed distinctly, since small variations of $\lambda$ are sufficient to cause large variations of $B$, and taking also into account the variations of $\phi$, the colours overlap so as to cause all trace of variation of intensity or colour to disappear.

Since $\mathrm{B}^{2}+\mathrm{C}^{2}$ is unchanged by the substitution of $-y$ for $y$, the rings are equally distinct on the two sides of the plane $x z$.

Again, since $\theta$ lies between 0 and $\frac{\pi}{z}$, when $\mu$ is greater than unity (i.e. in general) $z_{1}$ is positive. Hence

$$
\left(x^{2}+y^{2}\right) \sin \theta+2 x z_{1}
$$

is decreased by the substitution of $-x$ for $x$.

Thus the part of the rings on the side of the plane $y z$ which is nearest the incident light is less distinct than the other part.

With the help of the equation of the "surface of interference," we find that 


$$
\sqrt{\mathrm{B}^{2}+\mathrm{C}^{2}}=\frac{2 y \cos \theta\left(z+z_{1}\right)}{x} \sqrt{x^{2}+y^{2} \cos ^{2} \theta} .
$$

This vanishes when $\left(x^{2}+y^{2} \cos ^{2} \theta\right) / x$ vanishes.

Now

$$
\begin{aligned}
2\left(x^{2}+y^{2} \cos ^{2} \theta\right)\left(z+z_{1}\right) & =x\left[\left(x^{2}+y^{2}\right) \sin \theta+2 x z_{1}\right] \\
& =x\left[a^{2} \sin \theta+2 x z_{1}\right],
\end{aligned}
$$

$\therefore \sqrt{\mathrm{B}^{2}+\mathrm{C}^{\mathbf{2}}}$ vanishes when

$$
x=-\frac{a^{2} \sin \theta}{2 z_{1}}
$$

and since $x^{2}+y^{2}=a^{2}$, this requires that $x \ngtr a$,

$$
\begin{array}{cc}
\text { i.e. } & \frac{a \sin \theta}{2 z_{1}}<1, \\
\text { or } & h<\frac{4 z_{1}^{2}}{\lambda r} \frac{\cos \theta}{\sin ^{2} \theta} \\
\text { i.e. } & h<\frac{4 d^{2}}{\lambda r} \frac{\left(\mu^{2}-1\right)^{2} \sin ^{2} \theta \cos \theta}{\mu^{6} \cos ^{6} \theta_{1}}
\end{array}
$$

For all rings of lower order than the limit so determined there are two points quite distinctly visible, symmetrically situated with regard to the plane $x z$, and on the side of thy plane $y z$ farthest from the incident light.

To obtain the required criterion for bright rings it is only necessary to write $h+\frac{1}{2}$ for $h$ in the above.

If the rings are examined by means of a microscope, the breadth of the visual pencil is much greater than if the naked eye were used. Thus much greater values of $\phi$ are possible, and the obscurity is consequently increased.

For purposes of exact measurement, however, a microscope is necessary, in spite of the consequent greater indistinctness in the phenomena observed.

\section{Part II.-Newton's Rings in Transmitted Light.}

Section I. Calculation of the Relative Retardations.

We now proceed to consider the case of transmitted light. We shall take the axis of $z$ in the opposite direction from that before used in Part I., viz. drawn towards the side on which the lens lies (fig. 6). 
Fig. 6 .

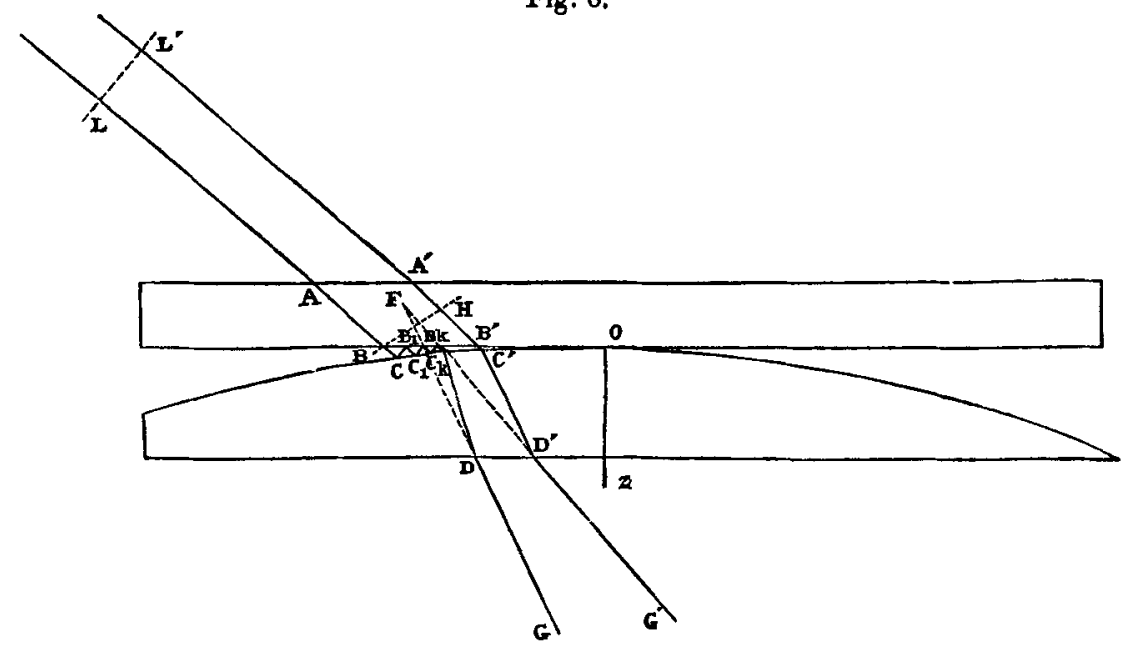

The direction-cosines will be similar to those used in Sect. II. Part I., so far as the two cases coincide, viz.:-

The direction-cosines

$$
\begin{aligned}
& \text { of } \mathrm{LA}, \mathrm{L}^{\prime} \mathrm{A}^{\prime}, \mathrm{BC}, \mathrm{B}^{\prime} \mathrm{C}^{\prime} \text { are }-l,-m, n \text {; } \\
& \text { of } \mathrm{AB}, \mathrm{A}^{\prime} \mathrm{B}^{\prime} \quad \text { are }-l^{\prime},-m^{\prime}, n^{\prime} \text {; } \\
& \text { of } \mathrm{B}_{1} \mathrm{C} \text { are } l_{1}, m_{1}, n_{1} \text {; of } \mathrm{B}_{1} \mathrm{C}_{1} \text { are }-l_{1},-m_{1} n_{1} \text {; } \\
& \text { \&c. \&c. } \\
& \text { of } \mathrm{B}_{k}, \mathrm{C}_{k-1} \text {, are } l_{k}, m_{k}, n_{k} ; \text { of } \mathrm{B}_{k}, \mathrm{C}_{k} \text { are }-l_{k},-m_{k}, n_{k} \text {. }
\end{aligned}
$$

Also we shall take as direction-cosines of

$$
\begin{aligned}
& \mathrm{C}_{k} \mathrm{D},-l_{k+1},-m_{k+1}, n_{k+1} ; \mathrm{C}^{\prime} \mathrm{D}^{\prime},-\alpha^{\prime},-\beta^{\prime}, \gamma^{\prime} ; \\
& \mathrm{FD},-l_{k+2},-m_{k+2}, n_{k+2} ; \mathrm{FD}^{\prime},-\alpha,-\beta, \gamma .
\end{aligned}
$$

The rectangular and spherical coordinates of

$\mathrm{C}$ are $\xi_{1}, \eta_{1}, \xi_{1}$, and $\phi, \psi$;

$\mathrm{C}_{1}$ are $\xi_{2}, \eta_{2}, \zeta_{2}$, and $\phi, \psi_{1}$;

$\& c$.

$\mathrm{C}_{k}$ are $\xi_{k+1}, \eta_{k+1}, \zeta_{k+1}$, and $\phi_{k}, \psi_{k}$;

$\mathrm{C}^{\prime}$ are $\xi^{\prime}, \eta^{\prime}, \zeta^{\prime}$, and $\phi^{\prime}, \psi^{\prime}$;

while $F$ is $\xi, \eta, \zeta$.

The thickness of the lens at its centre will be denoted by $d$, while $r$ is the radius of its convex surface. The direction. 
cosines of the normal at $\phi, \psi$ are

We have

$$
\sin \phi \cos \psi, \quad \sin \phi \sin \psi, \quad-\cos \phi .
$$

$$
\begin{aligned}
l_{\mathbf{L}}=l-2 h n \phi \cos \psi & +h(h-1) l \phi^{2} \\
& -2 h^{2} \phi \cos \psi[l \phi \cos \psi+m \phi \sin \psi] \\
m_{\mathbf{\Lambda}}=m-2 h n \phi \cos \psi+ & h(h-1) m \phi^{2} \\
& -2 h^{2} \phi \cos \psi[l \phi \cos \psi+m \phi \sin \psi] \\
n_{\mathbf{L}}=n+2 h(l \phi \cos \psi+ & m \phi \sin \psi)-h(h-1) \frac{1-n^{2}}{n} \phi^{2} \\
& +2 \frac{h^{2}}{n}[l \phi \cos \psi+m \phi \sin \psi]^{2}-2 l^{2} n \phi^{2} \\
\frac{1}{n_{k}}=\frac{1}{n}\left[1-\frac{2 h}{n}(l \phi \cos \psi+\right. & m \phi \sin \psi)+h(h-1) \frac{1-n^{2}}{n^{2}} \phi^{2} \\
& \left.+8 h^{2}\left(\frac{l \phi \cos \psi+m \phi \sin \psi}{n}\right)^{2}+2 h^{2} \phi^{2}\right]
\end{aligned}
$$

Putting

$$
\left.\begin{array}{rl}
\mathrm{U} & =l \sin \phi^{\prime} \cos \psi^{\prime}+m \sin \phi^{\prime} \sin \psi^{\prime}+n \cos \phi^{\prime} \\
& =n+l \phi^{\prime} \cos \psi^{\prime}+m \phi^{\prime} \sin \psi^{\prime}-n \frac{\phi^{\prime 2}}{2}+\ldots, \\
\alpha^{\prime} & =-\frac{1}{\mu}\left[\sin \phi^{\prime} \cos \psi^{\prime}\left\{\sqrt{\mu^{2}-1+\mathrm{U}^{2}}-\mathrm{U}\right\}+l\right], \\
\beta^{\prime} & =-\frac{1}{\mu}\left[\sin \phi^{\prime} \sin \psi^{\prime}\left\{\sqrt{\mu^{2}-1+\mathrm{U}^{2}}-\mathrm{U}\right\}+m\right], \\
\boldsymbol{\gamma}^{\prime} & =+\frac{1}{\mu}\left[\cos \phi^{\prime}\left\{\sqrt{\mu^{2}-1+\mathrm{U}^{2}}-\mathrm{U}\right\}+n\right] .
\end{array}\right\}
$$

And

$$
a=\mu \alpha^{\prime}, \quad \beta=\mu \beta^{\prime}, \quad \gamma=\sqrt{1-\mu^{2}+\mu^{2} \gamma^{\prime 2}} ;
$$

$\therefore \alpha=l-\left(n-\mu n^{\prime}\right) \phi^{\prime} \cos \psi^{\prime}-\left(1-\frac{n}{\mu n^{\prime}}\right) \phi^{\prime} \cos \psi^{\prime}$

$$
\left(l \phi^{\prime} \cos \psi^{\prime}+m \phi^{\prime} \sin \psi^{\prime}\right) \text {, }
$$

$$
\beta=m-\left(n-\mu n^{\prime}\right) \phi^{\prime} \sin \psi^{\prime}-\left(1-\frac{n}{\mu n^{\prime}}\right) \phi^{\prime} \sin \psi^{\prime}
$$

$$
\left(l \phi^{\prime} \cos \psi^{\prime}+m \phi^{\prime} \sin \psi^{\prime}\right) \text {; }
$$

$\mu^{2} \gamma^{\prime 2}=\mu^{2}-1+n^{2}-2\left(\mu n^{\prime}-n\right)\left(l \phi^{\prime} \cos \psi^{\prime}+m \phi^{\prime} \sin \psi^{\prime}\right)$

$$
-\left(\mu n^{\prime}-n\right)^{2} \phi^{\prime 2}+2 \frac{\mu n^{\prime}-n}{\mu n^{\prime}}\left(l \phi^{\prime} \cos \psi^{\prime}+m \phi^{\prime} \sin \psi^{\prime}\right)^{2} .
$$


Again,

Therefore, writing

$$
\begin{aligned}
& \xi-\frac{\alpha}{\gamma}(d-\zeta)+\frac{\alpha^{\prime}}{\gamma^{\prime}}\left(d-\zeta^{\prime}\right)=\xi^{\prime}=r \phi^{\prime} \cos \psi^{\prime}, \\
& \eta-\frac{\beta}{\gamma}(d-\zeta)+\frac{\beta^{\prime}}{\gamma^{\prime}}\left(d-\zeta^{\prime}\right)=\eta^{\prime}=r \phi^{\prime} \sin \psi^{\prime} .
\end{aligned}
$$

$$
u=\xi+\frac{\alpha}{\gamma}\left(\xi-\delta_{1}\right), v=\eta+\frac{\beta}{\gamma}\left(\zeta-\delta_{1}\right), \ldots
$$

where

we have

$$
\delta_{1}=d\left[1-\frac{\tilde{\gamma}}{\mu \gamma^{\prime}}\right], \quad \delta_{2}=d\left[1-\frac{\gamma^{3}}{\mu^{2} \gamma^{\prime 3}}\right],
$$

$$
\left.\begin{array}{c}
u-\frac{\alpha}{\mu \gamma^{\prime}} \zeta^{\prime}=r \phi^{\prime} \cos \psi^{\prime}, \\
v-\frac{\beta}{\mu \gamma^{\prime}} \zeta^{\prime}=r \phi^{\prime} \sin \psi^{\prime} . \\
\therefore r^{2} \phi^{\prime 2}=u^{2}+v^{2}-2 \frac{\alpha u+\beta v}{\mu \gamma^{\prime}} \zeta^{\prime} ; \\
\therefore \zeta^{\prime}=\frac{r \phi^{\prime 2}}{2}=\frac{1}{2} \frac{u^{2}+v^{2}}{r}-\frac{1}{2} \frac{\alpha u+\beta v}{\mu \gamma^{\prime}} \frac{u^{2}+v^{2}}{r^{2}} ; \\
\therefore \phi^{\prime} \cos \psi^{\prime}=\frac{u}{r}-\frac{1}{2} \frac{\alpha}{\mu \gamma^{\prime}} \frac{u^{2}+v^{2}}{r^{2}}+\frac{1}{2} \frac{\alpha}{\mu^{2} \gamma^{12}} \frac{\alpha u+\beta v}{r} \frac{u^{2}+v^{2}}{r^{2}}, \\
\phi^{\prime} \sin \psi^{\prime}=\frac{v}{r}-\frac{1}{2} \frac{\beta}{\mu \gamma^{\prime}} \frac{u^{2}+v^{2}}{r^{2}}+\frac{1}{2} \frac{\beta}{\mu^{2} \gamma^{\prime 2}} \frac{\alpha u+\beta v}{r} \frac{u^{2}+v^{2}}{r^{2}} .
\end{array}\right\}
$$

Wo shall now proceed to connect $\phi, \psi$ with $\phi^{\prime}, \psi^{\prime}$. Writing

$$
\begin{array}{ll}
\delta_{1}^{\prime}=d\left[1-\frac{n}{\mu n^{\prime}}\right], & \delta_{2}^{\prime}=d\left[1-\frac{n^{3}}{\mu^{3} n^{13}}\right], \\
u_{1}=\xi+\frac{l}{n}\left(\zeta-\delta_{1}^{\prime}\right), & v_{1}=\eta+\frac{m}{n}\left(\zeta-\delta_{1}^{\prime}\right), \\
\epsilon_{h}=h-\frac{\mu \gamma^{\prime}}{\gamma}, & \epsilon_{h}^{\prime}=l_{l}-\frac{\mu n^{\prime}}{n} .
\end{array}
$$

Now $l_{k+2}, m_{k+2}, n_{k+2}$ are derived from $l_{k}, m_{k}, n_{k}$ as $\alpha, \beta, \gamma$ are from $l, m, n, \phi^{\prime} \psi^{\prime}$ being replaced by $\phi_{k} \psi_{k}$.

Now

$$
\begin{aligned}
& \phi_{k} \cos \psi_{k}=\phi \cos \psi-k \frac{l}{n} \phi^{2}, \\
& \phi_{k} \sin \psi_{k}=\phi \sin \psi-k \frac{m}{n} \phi^{2} .
\end{aligned}
$$


Therefore

$$
l_{k+2}=l_{k}-\left(n_{k}-\mu n_{k}^{\prime}\right) \phi_{k} \cos \psi_{k}
$$

$-\left(1-\frac{n_{k}}{\mu n_{k}^{\prime}}\right) \phi_{k} \cos \psi_{k}\left[l_{k} \phi_{\dot{k}} \cos \psi_{k}+m_{k} \phi_{k} \sin \psi_{k}\right]$,

$$
\begin{aligned}
=l-n \epsilon_{2 k+1}^{\prime} \phi \cos \psi+k \epsilon_{k}^{\prime} l \phi^{2}-\left[2 k^{2}+2 k+1\right. \\
\left.-(2 k+1) \frac{n}{\mu n^{\prime}}\right] \phi \cos \psi(l \phi \cos \psi+m \phi \sin \psi),
\end{aligned}
$$

$n i_{k+2}=m-n \epsilon_{2 k+1}^{\prime} \phi \sin \psi+k \epsilon_{k}^{\prime} m \phi^{2}-\left[2 k^{2}+2 k+1\right.$

$$
\left.-(2 k+1) \frac{n}{\mu n^{\prime}}\right] \phi \cos \psi(l \phi \cos \psi+m \phi \sin \psi),
$$

$n_{k+2}^{2}=n^{2}+2 n \epsilon_{2 k+1}^{\prime}(l \phi \cos \psi+m \phi \sin \psi)-2 k \epsilon_{k}^{\prime}\left(1-n^{2}\right) \phi^{2}+2\left[2 k^{2}\right.$

$\left.+2 k+1-\overline{2 k+1} \frac{n}{\mu n^{\prime}}\right](l \phi \cos \psi+m \phi \sin \psi)^{q}-n^{2} \epsilon^{\prime 2}{ }_{2 k+1} \phi^{2}$,

$$
l_{k+2}=\mu l_{k+1}, \quad m_{k+2}=\mu m_{k+1}, \quad \mu n_{k+1}=\sqrt{\mu^{2}-1+n_{k+2}^{2}} .
$$

\section{Therefore}

$$
\begin{aligned}
& \frac{1}{n_{k+2}}=\frac{1}{n}\left[1-\epsilon_{2 k+1}^{\prime} \frac{l \phi \cos \psi+m \phi \sin \psi}{n}+k \epsilon_{k}^{\prime} \frac{1-n^{2}}{n^{2}} \phi^{2}+\frac{1}{2} \epsilon^{\prime k_{2 k+1}} \phi^{2}\right. \\
& \left.+\left[\frac{3}{2} k^{2{ }_{2 k+1}}-\left[2 k^{2}+2 k+1-\overline{2 k+1} \frac{n}{\mu n^{\prime}}\right]\right]\left(\frac{l \phi \cos \psi+m \phi \sin \psi}{n}\right)^{2}\right] \\
& \frac{1}{n_{k+1}}=\frac{1}{n^{\prime}}\left[1-\frac{n \epsilon_{2 k+1}^{\prime}}{\mu^{2} n^{\prime 2}}+k \epsilon_{k}^{\prime} \frac{1-n^{2}}{\mu^{2} n^{12}} \phi^{2}+\frac{1}{2} \frac{n^{2}}{\mu^{2} n^{12}} \epsilon^{\prime 2}{ }_{2 k+1} \phi^{2}+\frac{1}{\mu^{2} n^{12}}\left[\frac{3}{2} \frac{n^{2} \epsilon_{2 k+1}^{\prime 2}}{\mu^{2} n^{\prime 2}}\right.\right. \\
& \left.\left.\left.-\left(2 k^{2}+2 k+1-\overline{2 k+1} \frac{n}{\mu n^{\prime}}\right)\right] l \phi \cos \psi+m \phi \sin \psi\right)^{2}\right] \text {. }
\end{aligned}
$$

The values of $l, m, n$ in terms of $\alpha, \beta, \gamma$ may be deduced from those of $\alpha, \beta, \gamma$ in terms of $l, m, n$.

They are:-

$$
\left.\begin{array}{c}
l=\alpha+\gamma \epsilon_{1} \frac{u}{r}+\frac{1}{2} \alpha\left[1-\frac{\gamma}{\mu \gamma^{\prime}}\right] \frac{u^{2}+v^{2}}{r^{2}}-\epsilon_{1} \frac{u}{r} \frac{\alpha u+\beta v}{r} ; \\
m=\beta+\gamma \epsilon_{1} \frac{v}{r}+\frac{1}{2} \beta\left[1-\frac{\gamma}{\mu \gamma^{\prime}}\right] \frac{u^{2}+v^{2}}{r^{2}}-\epsilon_{1} \frac{v}{r} \frac{\alpha u+\beta v}{r} ; \\
n=\gamma-\epsilon_{1} \frac{\alpha u+\beta v}{r}-\frac{1}{2} \frac{1-\gamma^{2}}{\gamma}\left(1-\frac{\gamma}{\mu \gamma^{\prime}}\right) \frac{u^{2}+v^{2}}{r^{2}} \\
+\frac{\epsilon_{1}}{\gamma}\left(\frac{\alpha u+\beta v}{r}\right)^{2}-\frac{1}{2} \gamma \epsilon_{1}^{2} \frac{u^{2}+v^{2}}{r^{2}}-\frac{1}{2} \frac{\epsilon_{1}^{2}}{\gamma}\left(\frac{\alpha u+\beta v}{r}\right)^{2} . \\
\mu n^{\prime}=\mu \gamma^{\prime}-\epsilon_{1} \frac{\gamma}{\mu \gamma^{\prime}} \frac{\alpha u+\beta v}{r}-\& c .
\end{array}\right\}
$$




$$
\begin{aligned}
\therefore \delta_{1}^{\prime} & =d\left[1-\frac{n}{\mu n^{\prime}}\right]=\delta_{1}+\epsilon_{1} \frac{\alpha u+\beta v}{\gamma r}\left(\delta_{2}-\delta_{1}\right) ; \\
\therefore u_{1} & =\xi+\frac{l}{n}\left(\zeta-\delta_{1}{ }^{\prime}\right) ; \\
\therefore \frac{u_{1}}{r} & =\frac{u}{r}+\epsilon_{1} \frac{u}{r}\left(\zeta-\delta_{1}\right)+\frac{\alpha}{\gamma^{2}} \epsilon_{1} \frac{\alpha u+\beta v}{r}\left(\zeta-\delta_{2}\right), \\
& \frac{v_{1}}{r}=\frac{v}{r}+\epsilon_{1} \frac{v}{r}\left(\zeta-\delta_{1}\right)+\frac{\beta}{\gamma^{2}} \epsilon_{1} \frac{\alpha u+\beta v}{r}\left(\zeta-\delta_{2}\right) .
\end{aligned}
$$

Again,

$r \phi \cos \psi=\xi_{1}=\xi-\frac{l_{k+2}}{n_{k+2}}(d-\zeta)+\frac{l_{k+1}}{n_{k+1}}\left(d-\zeta_{k+1}\right)+\sum_{h=1}^{k=k} \frac{l_{h}}{n_{h}}\left(\zeta_{h}+\zeta_{h+1}\right)$,

$r \phi \sin \psi=\eta_{1}=\eta-\frac{m_{k+2}}{n_{k+2}}(d-\zeta)+\frac{m_{k+1}}{n_{k+1}}\left(d-\zeta_{k+1}\right)+\sum_{h=1}^{h=k} \frac{m_{h}}{n_{h}}\left(\zeta_{h}+\zeta_{h+1}\right)$.

Using the values already found in (v.) for the directioncosines, we have

$$
\begin{aligned}
& \phi \cos \psi= \frac{u_{1}}{r}-\epsilon_{2 k+1}^{\prime} \frac{u \zeta-\delta_{1}^{\prime}}{r}-\frac{l}{n^{2}} \epsilon_{2 k+1}^{\prime} \frac{l u_{1}+m v_{1}}{r} \frac{\zeta-\delta_{2}^{\prime}}{r} \\
&+\frac{1}{2} \frac{l}{n}\left[2 k-\frac{n}{\mu n^{\prime}}\right] \frac{u_{1}^{2}+v_{1}^{8}}{r} \\
&=\frac{u}{r}-2 k \frac{u}{r} \frac{\zeta-\delta_{1}}{r}-2 k \frac{\alpha}{\gamma^{2}} \frac{a u+\beta v}{r} \frac{\zeta-\delta_{2}}{r}+\frac{1}{2} \frac{\alpha}{\gamma}\left[2 k-\frac{\gamma}{\mu \gamma}\right] \frac{u^{2}+v^{2}}{r^{2}} ;
\end{aligned}
$$

$\phi \sin \psi=\frac{v}{r}-2 k \frac{v}{r} \frac{\zeta-\delta_{1}}{r}-2 k \frac{\beta}{\gamma^{2}} \frac{\alpha u+\beta v}{r} \frac{\zeta-\delta_{2}}{r}+\frac{1}{2} \frac{\beta}{\gamma}\left[2 k-\frac{\gamma}{\mu \gamma^{\prime}}\right] \frac{u^{2}+v^{2}}{r^{2}}$.

And

$$
\begin{gathered}
\phi_{k}^{2}=\phi^{2}-2 h \frac{\alpha u+\beta v}{\gamma r} \frac{u^{2}+v^{2}}{r^{2}} \\
=\frac{u^{2}+v^{2}}{r^{2}}-4 k \frac{u^{2}+v^{2}}{r^{2}} \frac{\zeta-\delta_{1}}{r}-4 k\left(\frac{\alpha u+\beta v}{\gamma r}\right)^{2} \frac{\zeta-\delta_{2}}{r} \\
+\left[2 k-\frac{\gamma}{\mu \gamma^{\prime}}\right] \frac{\alpha u+\beta v}{\gamma r} \frac{u^{2}+v^{2}}{r^{2}}-2 h \frac{\alpha u+\beta v}{\gamma r} \frac{u^{2}+v^{2}}{r^{2}} .
\end{gathered}
$$

and

$$
\phi^{2} \text { is obtained by putting } h=0
$$

$\phi^{\prime 2}$ is obtained by putting $k=0$ as well as $h=0$.

If a plane be drawn through $B$ perpendicular to $\mathrm{B}^{\prime} \mathrm{A}^{\prime}$ and $\mathrm{BA}$, and cutting the former in $\mathrm{H}$, the points $\mathrm{B}, \mathrm{H}$ are in the same wave-front which is incident in the direction LA. 
Also the coordinates of $B^{\prime}$ are :-

$$
\begin{aligned}
& \xi-\frac{\alpha}{\gamma}(d-\zeta)+\frac{\alpha^{\prime}}{\gamma^{\prime}}\left(d-\zeta^{\prime}\right)+\frac{l}{\eta} \zeta^{\prime}, \\
& \eta-\frac{\beta}{\gamma}(d-\zeta)+\frac{\beta^{\prime}}{\gamma^{\prime}}\left(d-\zeta^{\prime}\right)+\frac{m}{n} \zeta^{\prime} ;
\end{aligned}
$$

and those of $B$ are :-

$$
\xi_{1}+\frac{l}{n} \zeta_{1}, \quad \eta_{1}+\frac{m}{n} \zeta_{1}
$$

$\xi_{1}, \eta_{1}$ being given in (viii.). $F$ is

The difference of path (measured in air) of the two rays, at

$$
\begin{gathered}
\frac{1}{n} \zeta_{t}+\sum_{k=1}^{h=k}-\frac{1}{n_{h}}\left(\zeta_{h}+\zeta_{h+1}\right)+\mu \frac{d-\zeta_{k+1}}{n_{k+1}}+\frac{d-\zeta}{n_{k+2}} \\
-\left[\frac{1}{n} \zeta^{\prime}+\mu \frac{d-\zeta^{\prime}}{\gamma^{\prime}}-\frac{d-\zeta}{\gamma}+\mu \mathrm{B}^{\prime} \mathrm{H}\right] \\
=\frac{1}{n}\left[\zeta_{1}-\zeta^{\prime}\right]-\sum_{k=1}^{h=k} \frac{l l_{h}+m m_{h}-1}{n_{h}}\left[\zeta_{h}+\zeta_{h+1}\right] \\
\quad+\frac{l l_{k+2}+m m_{k+2}-1}{n_{k+2}}(d-\zeta) \\
-\frac{l l^{k+1}+m m_{k+1}-\mu}{n_{k+1}}\left(d-\zeta_{k+1}\right)-\frac{l a+m \beta-1}{\gamma}(d-\zeta) \\
\quad+\frac{l \alpha^{\prime}+m \beta^{\prime}-\mu}{\gamma^{\prime}}\left(d-\zeta^{\prime}\right)+\frac{1-n^{2}}{n}\left(\zeta^{\prime}-\zeta_{1}\right) .
\end{gathered}
$$

Now

and

$$
\frac{l l_{h}+m m_{h}-1}{n_{k}}=-n+2 \text { nd order terms, }
$$

$$
\zeta_{h}+\zeta_{h+1}=\frac{r}{2}\left[\phi_{h-1}^{2}+\phi_{h}{ }^{2}\right]=r \phi^{2}-(2 h-1) \frac{\alpha u+\beta v}{\gamma r} \frac{u^{2}+v^{2}}{r} ;
$$

i. e. is of the 2 nd order, and therefore we need not calculate the $2 \mathrm{nd}$ order terms in $\frac{l l_{h}+m m_{n}-1}{n_{k}}$.

Evaluating the other terms by means of equations (iv.) (v.) (vi.) (ix.), we obtain the following result:-

$$
\begin{aligned}
& \Delta_{k}=k \gamma \frac{u^{2}+v^{2}}{r}+k \frac{\mu^{2}-1}{\mu \gamma \gamma^{\prime}} \frac{\alpha u+\beta v}{r} \frac{u^{2}+v^{2}}{r} \\
& -\frac{k^{2} \gamma}{r^{2}}\left[2\left(u^{2}+v^{2}\right)\left(\zeta-\delta_{1}\right)+2\left(\frac{\alpha u+\beta v}{\gamma}\right)^{2}\left(\zeta-\delta_{2}\right)-\frac{\alpha u+\beta v}{\gamma}\left(u^{2}+v^{2}\right)\right]
\end{aligned}
$$

as given by Herr Gumlich in Wiedemann's Annalen, xxvi. p. 361 . 
Section II. The Shape of the Rings.

The only difference between this expression and that of equation (viii.) of Sect. II. Part I. is in the 2nd term, $\alpha, \beta, \gamma$ taking the place of $l, m, n$.

It is evident that the same process as that adopted in the former part of the paper will give similar results.

Putting

$$
\sin \theta=\alpha, \quad 0=\beta, \quad \cos \theta=\gamma,
$$

( $\theta$ is the angle of emergence)

the equation of the "surface of interference," referred to a system of axes of which the $z$-axis is in the direction of emergence, and the substitutions are those of Sect. III. Part I., is

$$
\begin{aligned}
& x\left(x^{2}+y^{2}\right) \sin \theta-2 x^{2} z-2 y^{2} \cos ^{2} \theta\left(z+z_{1}\right)=0 . \\
\therefore \Delta_{k}= & \frac{k \cos \theta}{r}\left(x^{2}+y^{2}\right)+k \frac{\mu^{2}-1}{\mu \cos \theta_{1}} x \frac{\left(x^{2}+y^{2}\right)}{r^{2}} \tan \theta \\
= & \frac{k}{r}\left(x^{2}+y^{2}\right) \cos \theta\left[1+\frac{x}{r} \frac{\mu^{2}-1}{\cos ^{2} \theta} \tan \theta_{1}\right] .
\end{aligned}
$$

The other equation to the rings is, therefore,

$$
\frac{x^{2}+y^{2}}{r} \cos \theta\left[1+\frac{x}{r} \frac{\left(\mu^{2}-1\right)}{\cos ^{2} \theta} \tan \theta_{1}\right]=h \lambda \text { or } \frac{2 h+1}{2} \lambda
$$

according as we consider the bright or dark rings.

Taking the former and putting $a^{2}$ for $\frac{h r \lambda}{\cos \theta}$, this becomes

$$
x^{2}+y^{2}=a^{2}\left[1-\frac{x}{r} \frac{\mu^{2}-1}{\cos ^{2} \theta} \tan \theta_{1}\right] . . .
$$

Thus in this case the rings lie on elliptic cylinders which are not co-axial.

The distance of the centre of the $l$ th bright ring from the central spot, from the origin, is

$$
\frac{1}{2} a^{2} \frac{x}{r} \frac{\mu^{2}-1}{\cos ^{2} \theta} \tan \theta_{1}=\frac{1}{2} h \lambda x \frac{\mu^{2}-1}{\cos ^{3} \theta} \tan \theta_{1} .
$$

This increases with $h$ and with $\lambda$, but is of the order $\lambda_{\frac{3}{3}}^{\frac{3}{3}}$ unless $\theta$ be nearly $\frac{\pi}{2}$, when these differences would become very perceptible. In ordinary cases the difference is not observable, and the rings are practically co-axial.

The shape of the surface of interference is exactly the same as that considered in the earlier part of this paper, and the conditions of distinctness remain unaltered.

December 1889. 